\title{
RICCI SOLITONS IN ALMOST $f$-COSYMPLECTIC MANIFOLDS
}

\author{
XIAOMIN CHEN
}

\begin{abstract}
In this article we study an almost $f$-cosymplectic manifold admitting a Ricci soliton. We first prove that there do not exist Ricci solitons on an almost cosymplectic $(\kappa, \mu)$-manifold. Further, we consider an almost $f$-cosymplectic manifold admitting a Ricci soliton whose potential vector field is the Reeb vector field and show that a three dimesional almost $f$-cosymplectic is a cosymplectic manifold. Finally we classify a three dimensional $\eta$-Einstein almost $f$-cosymplectic manifold admitting a Ricci soliton.
\end{abstract}

\section{INTRODUCTION}

A Ricci soliton is a Riemannian metric defined on manifold $M$ such that

$$
\frac{1}{2} \mathcal{L}_{V} g+R i c-\lambda g=0
$$

where $V$ and $\lambda$ are the potential vector field and some constant on $M$, respectively. Moreover, the Ricci soliton is called shrinking, steady and expanding according as $\lambda$ is positive, zero and negative respectively. The Ricci solitons are of interest to physicists as well and are known as quasi Einstein metrics in the physics literature [6. Compact Ricci solitons are the fixed point of the Ricci flow: $\frac{\partial}{\partial t} g=-2$ Ric, projected from the space of metrics onto its quotient modulo diffeomorphisms and scalings, and often arise as blow-up limits for the Ricci flows on compact manifolds. The study on the Ricci solitons has a long history and a lot of achievements were acquired, see [5, 13, 15] etc. On the other hand, the normal almost contact manifolds admitting Ricci solitons were also been studied by many researchers (see [3, 7, 8, 9]).

Recently, we note that the three dimensional almost Kenmotsu manifolds admitting Ricci solitons were considered $([18,19])$ and Cho ([2]) gave the classification of an almost cosymplectic manifold admitting a Ricci soliton whose potential vector field is the Reeb vector field. Here the almost cosymplectic manifold, defined by Goldberg and Yano [10, was an almost contact manifold whose 1-form $\eta$ and fundamental 2-form $\omega$ are closed, and the almost Kenmotsu manifold is an almost contact manifold satisfying $d \eta=0$ and $d \omega=2 \eta \wedge \omega$. Based on this Kim and Pak 11] introduced the concept of almost $\alpha$-cosymplectic manifold, i.e., an almost contact manifold satisfying $d \eta=0$ and $d \omega=2 \alpha \eta \wedge \omega$ for any real number $\alpha$. In particular, if $\alpha$ is non-zero it is said to be an almost $\alpha$-Kenmotsu manifold. Later Aktan et al. 1 defined an almost $f$-cosymplectic manifold $M$ by generalizing the real number $\alpha$ to a smooth function $f$ on $M$, i.e., an almost contact manifold satisfies $d \omega=2 f \eta \wedge \omega$ and $d \eta=0$ for a smooth function $f$ satisfying $d f \wedge \eta=0$. Clearly, an almost $f$-cosymplectic

2010 Mathematics Subject Classification. 53D15; 53C21; 53C25.

Key words and phrases. Ricci soliton; almost $f$-cosymplectic manifold; almost cosymplectic manifold; Einstein manifold; $(\kappa, \mu)$-manifold.

The author is supported by the Science Foundation of China University of PetroleumBeijing(No.2462015YQ0604) and partially by the Personnel Training and Academic Development Fund (2462015QZDX02). 
manifold is an almost cosymplectic manifold under the condition that $f=0$ and an almost $\alpha$-Kenmotsu manifold if $f$ is constant $(\neq 0)$. In particular, if $f=1$ then $M$ is an almost Kenmotsu manifold.

On the other hand, we observe that a remarkable class of contact metric manifold is $(\kappa, \mu)$-space whose curvature tensor satisfies

$$
R(X, Y) \xi=\kappa(\eta(Y) X-\eta(X) Y)+\mu(\eta(Y) h X-\eta(X) h Y)
$$

for any vector fields $X, Y$, where $\kappa$ and $\mu$ are constants and $h:=\frac{1}{2} \mathcal{L}_{\xi} \phi$ is a self-dual operator. In fact Sasakian manifolds are special $(\kappa, \mu)$-spaces with $\kappa=1$ and $h=0$. An almost cosymplectic $(\kappa, \mu)$-manifold is an almost cosymplectic manifold with curvature tensor satisfying (1.2). Endo proved that if $\kappa \neq 0$ any almost cosymplectic $(\kappa, \mu)$-manifolds are not cosymplectic (4). Furthermore, since $\kappa \phi^{2}=h^{2}, \kappa \leq 0$ and the equality holds if and only if the almost cosymplectic $(\kappa, \mu)$-manifolds are cosymplectic. Notice that Wang proved the non-existence of gradient Ricci solitons in almost cosymplectic $(\kappa, \mu)$-manifolds(see [17]).

In this paper we first obtain an non-existence of a Ricci soliton in almost cosymplectic $(\kappa, \mu)$-manifolds, namely

Theorem 1.1. There do not exist Ricci solitons on almost cosymplectic $(\kappa, \mu)$-manifolds with $\kappa<0$ and $-\frac{1}{2}<\mu<0$.

Next we consider a three-dimensional almost $f$-cosymplectic manifold admitting a Ricci soliton whose potential vector field is the Reeb vector field $\xi$, and prove the following theorem.

Theorem 1.2. A three-dimensional almost $f$-cosymplectic manifold $M$ admits a Ricci soliton whose potential vector field is $\xi$ if and only if $\xi$ is Killing and $M$ is Ricci flat.

As it is well known that a $(2 n+1)$-dimensional almost contact manifold $(M, \phi, \xi, \eta, g)$ is said to be $\eta$-Einstein if its Ricci tensor satisfies

$$
R i c=a g+b \eta \otimes \eta,
$$

where $a$ and $b$ are smooth functions. For a three-dimensional $\eta$-Einstein almost $f$-cosymplectic manifold $M$ with a Ricci soliton we prove the following result:

Theorem 1.3. Let $(M, \phi, \eta, \xi, g)$ be a three-dimensional $\eta$-Einstein almost $f$-cosymplectic manifold admitting a Ricci soliton. Then either $M$ is an $\alpha$-cosymplectic manifold, or $M$ is an Einstein manifold of constant sectional curvature $\frac{\lambda}{2}$ with $\lambda=-2 \xi(f)-2 f^{2}$.

Remark 1.1. Our theorem extends the Wang and Liu's result [18. In fact, when $f=1$ then $a=-2$ in view of Proposition 5.1] in Section 5. Thus it follows from (2.8) that trace $\left(h^{2}\right)=0$, i.e., $h=0$. So $M$ is also a Kenmotsu manifold of sectional curvature -1 .

In order to prove these conclusions, in Section 2 we recall some basic concepts and formulas. The proofs of theorems will be given in Section 3, Section 4 and Section 5, respectively.

\section{Some BASIC CONCEPTS AND RELATED RESUltS}

In this section we will recall some basic concepts and equations. Let $M^{2 n+1}$ be a $(2 n+1)$ dimensional Riemannian manifold. An almost contact structure on $M$ is a triple $(\phi, \xi, \eta)$, where $\phi$ is a $(1,1)$-tensor field, $\xi$ a unit vector field, $\eta$ a one-form dual to $\xi$ satisfying

$$
\phi^{2}=-I+\eta \otimes \xi, \eta \circ \phi=0, \phi \circ \xi=0 .
$$


A smooth manifold with such a structure is called an almost contact manifold. It is well-known that there exists a Riemannian metric $g$ such that

$$
g(\phi X, \phi Y)=g(X, Y)-\eta(X) \eta(Y),
$$

for any $X, Y \in \mathfrak{X}(M)$. It is easy to get from (2.1) and (2.2) that

$$
g(\phi X, Y)=-g(X, \phi Y), \quad g(X, \xi)=\eta(X) .
$$

An almost contact structure $(\phi, \xi, \eta)$ is said to be normal if the corresponding complex structure $J$ on $M \times \mathbb{R}$ is integrable.

Denote by $\omega$ the fundamental 2-form on $M$ defined by $\omega(X, Y):=g(\phi X, Y)$ for all $X, Y \in \mathfrak{X}(M)$. An almost $\alpha$-cosymplectic manifold ([11, 14]) is an almost contact metric manifold $(M, \phi, \xi, \eta, g)$ such that the fundamental form $\omega$ and 1-form $\eta$ satisfy $d \eta=0$ and $d \omega=2 \alpha \eta \wedge \omega$, where $\alpha$ is a real number. In particular, $M$ is an almost cosymplectic manifold if $\alpha=0$ and an almost Kenmotsu manifold if $\alpha=1$. In [1, a class of more general almost contact manifolds was defined by generalizing the real number $\alpha$ to a smooth function $f$. More precisely, an almost contact metric manifold is called an almost $f$-cosymplectic manifold if $d \eta=0$ and $d \omega=2 f \eta \wedge \omega$ are satisfied, where $f$ is a smooth function with $d f \wedge \eta=0$. In addition, a normal almost $f$-cosymplectic manifold is said to be an $f$ cosymplectic manifold.

Let $M$ be an almost $f$-cosymplectic manifold, we recall that there is an operator $h=\frac{1}{2} \mathcal{L}_{\xi} \phi$ which is a self-dual operator. The Levi-Civita connection is given by (see [1])

$$
2 g\left(\left(\nabla_{X} \phi\right) Y, Z\right)=2 f g(g(\phi X, Y) \xi-\eta(Y) \phi X, Z)+g(N(Y, Z), \phi X)
$$

for arbitrary vector fields $X, Y$, where $N$ is the Nijenhuis torsion of $M$. Then by a simple calculation, we have

$$
\operatorname{trace}(h)=0, \quad h \xi=0, \quad \phi h=-h \phi, \quad g(h X, Y)=g(X, h Y), \quad \forall X, Y \in \mathfrak{X}(M) .
$$

Write $A X:=\nabla_{X} \xi$ for any vector field $X$. Thus $A$ is a $(1,1)$-tensor of $M$. Using (2.4), a straightforward calculation gives

$$
A X=-f \phi^{2} X-\phi h X
$$

and $\nabla_{\xi} \phi=0$. By (2.3), it is obvious that $A \xi=0$ and $A$ is symmetric with respect to metric $g$, i.e., $g(A X, Y)=g(X, A Y)$ for all $X, Y \in \mathfrak{X}(M)$. We denote by $R$ and Ric the Riemannian curvature tensor and Ricci tensor, respectively. For an almost $f$-cosymplectic manifold $\left(M^{2 n+1}, \phi, \xi, \eta, g\right)$ the following equations were proved([1]):

$$
\begin{aligned}
& R(X, \xi) \xi-\phi R(\phi X, \xi) \xi=2\left[\left(\xi(f)+f^{2}\right) \phi^{2} X-h^{2} X\right], \\
& \left(\nabla_{\xi} h\right) X=-\phi R(X, \xi) \xi-\left[\xi(f)+f^{2}\right] \phi X-2 f h X-\phi h^{2} X, \\
& \operatorname{Ric}(\xi, \xi)=-2 n\left(\xi(f)+f^{2}\right)-\operatorname{trace}\left(h^{2}\right), \\
& \operatorname{trace}(\phi h)=0, \\
& R(X, \xi) \xi=\left[\xi(f)+f^{2}\right] \phi^{2} X+2 f \phi h X-h^{2} X+\phi\left(\nabla_{\xi} h\right) X,
\end{aligned}
$$

for any vector fields $X, Y$ on $M$.

\section{Proof of Theorem 1.1}

In this section we suppose that $(M, \phi, \xi, \eta, g)$ is an almost cosymplectic $(\kappa, \mu)$-manifold, i.e., the curvature tensor satisfies (1.2). In the following we always suppose $\kappa<0$. The 
following relations are provided(see [12, Eq.(3.22) and Eq.(3.23)]):

$$
\begin{aligned}
Q & =2 n \kappa \eta \otimes \xi+\mu h, \\
h^{2} & =\kappa \phi^{2},
\end{aligned}
$$

where $Q$ is the Ricci operator defined by $\operatorname{Ric}(X, Y)=g(Q X, Y)$ for any vectors $X, Y$. In particular, $Q \xi=2 n \kappa \xi$ because of $h \xi=0$.

In view of (3.1) and the Ricci soliton equation (1.1), we obtain

$$
\left(\mathcal{L}_{V} g\right)(Y, Z)=2 \lambda g(Y, Z)-2 \mu g(h Y, Z)-4 n \kappa \eta(Y) \eta(Z)
$$

for any vectors $Y, Z$. Since $\kappa, \mu$ are two real numbers and $\nabla_{X} \xi=A X$, differentiating (3.3) along any vector field $X$ provides

$$
\begin{aligned}
\left(\nabla_{X} \mathcal{L}_{V} g\right)(Y, Z)= & \nabla_{X}\left(\left(\mathcal{L}_{V} g\right)(Y, Z)\right)-\mathcal{L}_{V} g\left(\nabla_{X} Y, Z\right)-\mathcal{L}_{V} g\left(Y, \nabla_{X} Z\right) \\
= & -2 \mu g\left(\left(\nabla_{X} h\right) Y, Z\right)-4 n \kappa \nabla_{X}(\eta(Y)) \eta(Z)-4 n \kappa \eta(Y) \nabla_{X}(\eta(Z)) \\
& +4 n \kappa \eta\left(\nabla_{X} Y\right) \eta(Z)+4 n \kappa \eta(Y) \eta\left(\nabla_{X} Z\right) \\
= & -2 \mu g\left(\left(\nabla_{X} h\right) Y, Z\right)-4 n \kappa g(Y, A X) \eta(Z)-4 n \kappa \eta(Y) g(Z, A X) .
\end{aligned}
$$

Moreover, making use of the commutation formula (see [20]):

$$
\begin{aligned}
& \left(\mathcal{L}_{V} \nabla_{X} g-\nabla_{X} \mathcal{L}_{V} g-\nabla_{[V, X]} g\right)(Y, Z)= \\
& \quad-g\left(\left(\mathcal{L}_{V} \nabla\right)(X, Y), Z\right)-g\left(\left(\mathcal{L}_{V} \nabla\right)(X, Z), Y\right),
\end{aligned}
$$

we derive

$$
\left(\nabla_{X} \mathcal{L}_{V} g\right)(Y, Z)=g\left(\left(\mathcal{L}_{V} \nabla\right)(X, Y), Z\right)+g\left(\left(\mathcal{L}_{V} \nabla\right)(X, Z), Y\right) .
$$

It follows from (3.4) and (3.5) that

$$
\begin{aligned}
g\left(\left(\mathcal{L}_{V} \nabla\right)(Y, Z), X\right)= & \frac{1}{2}\left\{\left(\nabla_{Z} \mathcal{L}_{V} g\right)(Y, X)+\left(\nabla_{Y} \mathcal{L}_{V} g\right)(Z, X)-\left(\nabla_{X} \mathcal{L}_{V} g\right)(Y, Z)\right\} \\
= & -\mu\left\{g\left(\left(\nabla_{Z} h\right) Y, X\right)+g\left(\left(\nabla_{Y} h\right) Z, X\right)-g\left(\left(\nabla_{X} h\right) Y, Z\right)\right. \\
& -4 n \kappa g(Y, A Z) \eta(X)\} .
\end{aligned}
$$

Hence for any vector $Y$,

$$
\left(\mathcal{L}_{V} \nabla\right)(Y, \xi)=-\mu\left\{\left(\nabla_{\xi} h\right) Y+2 \kappa \phi Y\right\}
$$

by using (3.2) and (2.5) . Lie differentiating (3.7) along $V$ and making use of the identity([20]):

$$
\left(\mathcal{L}_{V} R\right)(X, Y) Z=\left(\nabla_{X} \mathcal{L}_{V} \nabla\right)(Y, Z)-\left(\nabla_{Y} \mathcal{L}_{V} \nabla\right)(X, Z),
$$

we obtain

$$
\begin{aligned}
\left(\mathcal{L}_{V} R\right)(X, \xi) \xi & =\left(\nabla_{X} \mathcal{L}_{V} \nabla\right)(\xi, \xi)-\left(\nabla_{\xi} \mathcal{L}_{V} \nabla\right)(X, \xi) \\
& =-2\left(\mathcal{L}_{V} \nabla\right)\left(\nabla_{X} \xi, \xi\right)-\left(\nabla_{\xi} \mathcal{L}_{V} \nabla\right)(X, \xi) \\
& \left.=2 \mu\left\{\left(\nabla_{\xi} h\right) \nabla_{X} \xi+2 \kappa \phi \nabla_{X} \xi\right\}+\mu\left\{\left(\nabla_{\xi} \nabla_{\xi} h\right) X\right)\right\} \\
& \left.=2 \mu\left\{\phi\left(\nabla_{\xi} h\right) h X+2 \kappa h X\right\}+\mu\left\{\left(\nabla_{\xi} \nabla_{\xi} h\right) X\right)\right\} \\
& =\mu\left\{2 \kappa \mu \phi^{2} X+(4 \kappa+\mu) h X\right\} .
\end{aligned}
$$

Here we have used the following fact: By (2.7), (3.2) and (2.1), we get $\nabla_{\xi} h=-\mu \phi h$, thus

$$
\nabla_{\xi} \nabla_{\xi} h+2 \phi\left(\nabla_{\xi} h\right) h=\mu h+2 \kappa \mu \phi^{2} .
$$


Lie differentiating $R(X, \xi) \xi=-\kappa \phi^{2} X+\mu h X$ (a consequence of Eq.(1.2) ) and recalling (3.9), we have

$$
\begin{aligned}
& \mu\left\{2 \kappa \mu \phi^{2} X+(4 \kappa+\mu) h X\right\}+R\left(X, \mathcal{L}_{V} \xi\right) \xi+R(X, \xi) \mathcal{L}_{V} \xi \\
& =-\kappa\left(\mathcal{L}_{V} \eta\right)(X) \xi-\kappa \eta(X) \mathcal{L}_{V} \xi+\mu\left(\mathcal{L}_{V} h\right) X
\end{aligned}
$$

Using (1.2) again gives

$$
\begin{aligned}
R(X, \xi) \mathcal{L}_{V} \xi & =R\left(X, \mathcal{L}_{V} \xi\right) \xi-\left[\kappa\left(g\left(X, \mathcal{L}_{V} \xi\right) \xi-\eta(X) \mathcal{L}_{V} \xi\right)\right. \\
& \left.+\mu\left(g\left(h X, \mathcal{L}_{V} \xi\right) \xi-\eta(X) h \mathcal{L}_{V} \xi\right)\right] .
\end{aligned}
$$

On the other hand, it follows from (3.3) that

$$
\left(\mathcal{L}_{V} \eta\right)(X)=\left(\mathcal{L}_{V} g\right)(X, \xi)+g\left(X, \mathcal{L}_{V} \xi\right)=(2 \lambda-4 n \kappa) \eta(X)+g\left(X, \mathcal{L}_{V} \xi\right) .
$$

Now substituting (3.11) and (3.12) into (3.10) and using (1.2), we obtain

$$
\begin{aligned}
& \mu\left\{2 \kappa \mu \phi^{2} X+(4 \kappa+\mu) h X\right\}+2 \kappa \eta\left(\mathcal{L}_{V} \xi\right) X+2 \mu \eta\left(\mathcal{L}_{V} \xi\right) h X \\
& \quad-\kappa g\left(X, \mathcal{L}_{V} \xi\right) \xi-\kappa \eta(X) \mathcal{L}_{V} \xi-\mu g\left(h X, \mathcal{L}_{V} \xi\right) \xi-\mu \eta(X) h \mathcal{L}_{V} \xi \\
& =-\kappa\left[(2 \lambda-4 n \kappa) \eta(X)+g\left(X, \mathcal{L}_{V} \xi\right)\right] \xi-\kappa \eta(X) \mathcal{L}_{V} \xi+\mu\left(\mathcal{L}_{V} h\right) X
\end{aligned}
$$

Furthermore, notice that the Ricci tensor equation (3.1) implies the scalar curvature $r=2 n \kappa$ and recall the following integrability formula (see [16, Eq.(5)]):

$$
\mathcal{L}_{V} r=-\Delta r-2 \lambda r+2\|Q\|^{2}
$$

for a Ricci soliton. By (3.1), (3.2) and the foregoing formula we thus obtain $\lambda=2 n \kappa-\mu^{2}$. Also, it follows from (3.3) that $g\left(\mathcal{L}_{V} \xi, \xi\right)=2 n \kappa-\lambda$. Therefore $g\left(\mathcal{L}_{V} \xi, \xi\right)=\mu^{2}$. Hence Equation (3.13) becomes

$$
\begin{aligned}
& \mu(4 \kappa+\mu) h X+2 \mu^{3} h X-\kappa \eta(X) \mathcal{L}_{V} \xi-\mu g\left(h X, \mathcal{L}_{V} \xi\right) \xi-\mu \eta(X) h \mathcal{L}_{V} \xi \\
& =-\kappa \eta(X) \mathcal{L}_{V} \xi+\mu\left(\mathcal{L}_{V} h\right) X .
\end{aligned}
$$

Letting $X \in \mathfrak{D}$ be an eigenvector of $h$ in the above formula and taking the inner product with $X$, we find

$$
\mu\left(4 \kappa+\mu+2 \mu^{2}\right)=0
$$

If $\mu \neq 0$ then $4 \kappa+\mu+2 \mu^{2}=0$, which implies $\mu<-\frac{1}{2}$ or $\mu>0$.

If $\mu=0$. From (3.4), (3.5) and (3.6), we have

$$
g(Y, A X) \eta(Z)+g(Z, A X) \eta(Y)=0
$$

since $\kappa<0$. Now putting $Z=\xi$ gives $g(Y, A X)=0$ for any vector fields $X, Y$ because $A \xi=0$. That means that $A X=0$ for any vector field $X$. From (2.5) with $f=0$, we get $h=0$. Clearly, it is impossible. Therefore we complete the proof.

\section{Proof of Theorem 1.2}

In this section we assume that $M$ is a three dimensional almost $f$-cosymplectic manifold and the potential vector field $V$ is the Reeb vector field. Before giving the proof, we need to prove the following lemma. 
Lemma 4.1. For any almost $f$-cosymplectic manifold the following formula holds:

$$
\begin{aligned}
\left(\mathcal{L}_{\xi} R\right)(X, \xi) \xi= & 2 \xi(f) \phi h X-2\left[f\left(\nabla_{\xi} h\right) \phi X+\left(\nabla_{\xi} h\right) h X\right] \\
& +[\xi(\xi(f))+2 f \xi(f)] \phi^{2} X+\phi\left(\nabla_{\xi} \nabla_{\xi} h\right) X .
\end{aligned}
$$

Proof. Obviously, $\mathcal{L}_{\xi} \eta=0$ because $A \xi=0$. Notice that for any vector fields $X, Y, Z$ the 1-form $\eta$ satisfies the following relation([20]):

$$
-\eta\left(\left(\mathcal{L}_{X} \nabla\right)(Y, Z)\right)=\left(\mathcal{L}_{X}\left(\nabla_{Y} \eta\right)-\nabla_{Y}\left(\mathcal{L}_{X} \eta\right)-\nabla_{[X, Y]} \eta\right)(Z) .
$$

Putting $X=\xi$ and using (2.5) yields

$$
\begin{aligned}
-\eta\left(\left(\mathcal{L}_{\xi} \nabla\right)(Y, Z)\right) & =\left(\mathcal{L}_{\xi}\left(\nabla_{Y} \eta\right)-\nabla_{Y}\left(\mathcal{L}_{\xi} \eta\right)-\nabla_{[\xi, Y]} \eta\right)(Z) \\
& =\nabla_{\xi}\left(\left(\nabla_{Y} \eta\right)(Z)\right)-\left(\nabla_{Y} \eta\right)\left(\mathcal{L}_{\xi} Z\right)-g(A([\xi, Y]), Z) \\
& =\nabla_{\xi} g(A Y, Z)-g(A Y,[\xi, Z])-g(A([\xi, Y]), Z) \\
& =g\left(\left(\nabla_{\xi} A\right) Y, Z\right)+2 g(A Y, A Z) .
\end{aligned}
$$

In view of (3.5), we obtain from (4.2) that

$$
\begin{aligned}
g\left(\left(\mathcal{L}_{\xi} \nabla\right)(X, \xi), Y\right) & =\left(\nabla_{X} \mathcal{L}_{\xi} g\right)(Y, \xi)-g\left(\left(\mathcal{L}_{\xi} \nabla\right)(X, Y), \xi\right) \\
& =-2 g(A X, A Y)+g\left(\left(\nabla_{\xi} A\right) X, Y\right)+2 g(A X, A Y) \\
& =\xi(f) g(\phi X, \phi Y)+g\left(\left(\nabla_{\xi} h\right) X, \phi Y\right) .
\end{aligned}
$$

That is,

$$
\left(\mathcal{L}_{\xi} \nabla\right)(X, \xi)=-[\xi(f)] \phi^{2} X-\phi\left(\nabla_{\xi} h\right) X .
$$

Obviously, for any vector field $X$, we know $\left(\mathcal{L}_{\xi} \nabla\right)(X, \xi)=\left(\mathcal{L}_{\xi} \nabla\right)(\xi, X)$ from (3.6). Therefore we compute the Lie derivative of $R(X, \xi) \xi$ along $\xi$ as follows:

$$
\begin{aligned}
\left(\mathcal{L}_{\xi} R\right)(X, \xi) \xi= & \left(\nabla_{X} \mathcal{L}_{\xi} \nabla\right)(\xi, \xi)-\left(\nabla_{\xi} \mathcal{L}_{\xi} \nabla\right)(X, \xi) \\
= & -\left(\mathcal{L}_{\xi} \nabla\right)\left(\nabla_{X} \xi, \xi\right)-\left(\mathcal{L}_{\xi} \nabla\right)\left(\xi, \nabla_{X} \xi\right)-\left(\nabla_{\xi} \mathcal{L}_{\xi} \nabla\right)(X, \xi) \\
= & 2[\xi(f)] \phi^{2} A X+2 \phi\left(\nabla_{\xi} h\right) A X-\left(\nabla_{\xi} \mathcal{L}_{\xi} \nabla\right)(X, \xi) \\
= & 2 \xi(f)\left(f \phi^{2} X+\phi h X\right)+2\left[-f\left(\nabla_{\xi} h\right) \phi X-\left(\nabla_{\xi} h\right) h X\right] \\
& +[\xi(\xi(f))] \phi^{2} X+\phi\left(\nabla_{\xi} \nabla_{\xi} h\right) X .
\end{aligned}
$$

Here we used $\nabla_{\xi} \phi=0$ and $\phi \nabla_{\xi} h=-\left(\nabla_{\xi} h\right) \phi$ followed from $h \phi+\phi h=0$.

Proof of Theorem 1.2. Now we suppose that the potential vector $V=\xi$ in the Ricci equation (1.1). Then for any $X \in \mathfrak{X}(M)$,

$$
-f \phi^{2} X-\phi h X+Q X=\lambda X
$$

Putting $X=\xi$ in (4.3), we have

$$
Q \xi=\lambda \xi
$$

Moreover, the above formula together (2.8) with $n=1$ leads to

$$
\operatorname{trace}\left(h^{2}\right)=-\lambda-2 f^{2}-2 \xi(f) \text {. }
$$

Since the curvature tensor of a 3-dimension Riemannian manifold is given by

$$
\begin{aligned}
R(X, Y) Z= & g(Y, Z) Q X-g(X, Z) Q Y+g(Q Y, Z) X-g(Q X, Z) Y \\
& -\frac{r}{2}\{g(Y, Z) X-g(X, Z) Y\},
\end{aligned}
$$


where $r$ denotes the scalar curvature. Putting $Y=Z=\xi$ in (4.6) and applying (4.3) and (4.4), we obtain

$$
R(X, \xi) \xi=\left(\frac{r}{2}+f-2 \lambda\right) \phi^{2} X+\phi h X
$$

Contracting the above formula over $X$ leads to $\operatorname{Ric}(\xi, \xi)=-r-2 f+4 \lambda$, which follows from (4.4) that

$$
r+2 f=3 \lambda
$$

Taking the Lie derivative of (4.7) along $\xi$ and using (4.8), we obtain

$$
\left(\mathcal{L}_{\xi} R\right)(X, \xi) \xi=2 h^{2} X+\phi\left(\mathcal{L}_{\xi} h\right) X
$$

since $\mathcal{L}_{\xi} \phi^{2}=2 \phi h+2 h \phi=0$ and $h=\frac{1}{2} \mathcal{L}_{\xi} \phi$. By Lemma 4.1 and (4.9), we get

$$
\begin{aligned}
& 2 \xi(f) \phi h X-2\left[f\left(\nabla_{\xi} h\right) \phi X+\left(\nabla_{\xi} h\right) h X\right] \\
& \quad+[\xi(\xi(f))+2 f \xi(f)] \phi^{2} X+\phi\left(\nabla_{\xi} \nabla_{\xi} h\right) X \\
& =2 h^{2} X+\phi\left(\mathcal{L}_{\xi} h\right) X .
\end{aligned}
$$

By virtue of (2.7) and (4.7), we have

$$
\left(\nabla_{\xi} h\right) X=\left(-\frac{\lambda}{2}-\xi(f)-f^{2}\right) \phi X+(1-2 f) h X-\phi h^{2} X .
$$

Making use of the above equation we further compute

$$
\begin{aligned}
\phi\left(\nabla_{\xi} \nabla_{\xi} h\right) X= & (-\xi \xi(f)-2 f \xi(f)) \phi^{2} X-2 \xi(f) \phi h X+(1-2 f) \phi\left(\nabla_{\xi} h\right) X \\
& +\left(\nabla_{\xi} h\right) h X+h\left(\nabla_{\xi} h\right) X .
\end{aligned}
$$

As well as via (2.5) we get

$$
\begin{aligned}
\phi\left(\mathcal{L}_{\xi} h\right) X & =\phi \mathcal{L}_{\xi}(h X)-\phi h([\xi, X]) \\
& =\phi\left(\nabla_{\xi} h\right) X+\phi(h A-A h) X \\
& =\phi\left(\nabla_{\xi} h\right) X-2 h^{2} X .
\end{aligned}
$$

Substituting (4.12) and (4.13) into (4.10), we derive

$$
-\left(\nabla_{\xi} h\right) h X+h\left(\nabla_{\xi} h\right) X=0 .
$$

Further, applying (4.11) in the formula (4.14), we get

$$
\left(-\lambda-2 f^{2}-2 \xi(f)\right) h X-h^{3} X=0 .
$$

Write $\beta:=-\lambda-2 f^{2}-2 \xi(f)$, then the above equation is rewritten as $h^{3} X=\beta h X$ for every vector $X$. Denote by $e_{i}$ and $\lambda_{i}$ the eigenvectors and the corresponding eigenvalues for $i=1,2,3$, respectively. If $h \neq 0$ then there is a nonzero eigenvalue $\lambda_{1} \neq 0$ satifying $\lambda_{1}^{3}=\beta \lambda_{1}$, i.e., $\lambda_{1}^{2}=\beta$. Since trace $(h)=0$ and trace $\left(h^{2}\right)=\beta$ by (4.4), we have $\sum_{i=1}^{3} \lambda_{i}=0$ and $\sum_{i=1}^{3} \lambda_{i}^{2}=\beta$. This shows $\lambda_{2}=\lambda_{3}=0$, which further leads to $\lambda_{1}=0$. It is a contradiction. Thus we have

$$
\beta=-\left(\lambda+2 f^{2}+2 \xi(f)\right)=0 \quad \text { and } \quad h=0 .
$$

On the other hand, taking the covariant differentiation of $Q \xi=\lambda \xi$ (see (4.4)) along arbitrary vector field $X$ and using (2.5), one can easily deduce

$$
\left(\nabla_{X} Q\right) \xi+Q\left(-f \phi^{2} X-\phi h X\right)=-\lambda\left(f \phi^{2} X+\phi h X\right) .
$$


Contracting this equation over $X$ and using (4.3), we derive

$$
\frac{1}{2} \xi(r)-2 f^{2}-\operatorname{trace}\left(h^{2}\right)=0 .
$$

By virtue of (4.4) and (4.8), the foregoing equation yields

$$
\xi(f)+\lambda=0 .
$$

This shows that $\xi(f)$ is constant, then it infers from (4.15) that $f=0$ and $\lambda=0$, that means that $M$ is cosymplectic. Therefore it follows from (2.5) that $\left(\mathcal{L}_{\xi} g\right)(X, Y)=2 g(A X, Y)=0$ for any vectors $X, Y$. By (4.3), it is obvious that $Q=0$. Thus we complete the proof of Theorem 1.2 .

By the proof of Theorem 1.2 the following result is clear.

Corollary 4.1. A three-dimensional almost $\alpha$-Kenmotsu manifold $(M, \phi, \eta, \xi, g)$ does not admit a Ricci soliton with potential vector field being $\xi$.

\section{Proof of Theorem 1.3}

In this section we assume that $M$ is a three dimensional $\eta$-Einstein almost $f$-cosymplectic manifold, i.e., the Ricci tensor Ric $=a g+b \eta \otimes \eta$. We first prove the following proposition.

Proposition 5.1. Let $M$ be a three dimensional $\eta$-Einstein almost $f$-cosymplectic manifold. Then the following relation is satisfied:

$$
2 \xi(f)+2 f^{2}+(a+b)=0 .
$$

Proof. Since $M$ is $\eta$-Einstein, we know that $Q \xi=(a+b) \xi$ and the scalar curvature $r=3 a+b$. Hence it follows from (4.6) that

$$
R(X, \xi) \xi=-\frac{a+b}{2} \phi^{2} X .
$$

By Lie differentiating (5.1), we derive

$$
\left(\mathcal{L}_{\xi} R\right)(X, \xi) \xi=-\frac{\xi(a+b)}{2} \phi^{2} X .
$$

Also, it follows from (5.1) and (2.7) that

$$
\left(\nabla_{\xi} h\right) X=-\left[\xi(f)+f^{2}+\frac{1}{2}(a+b)\right] \phi X-2 f h X-\phi h^{2} X .
$$

Moreover, we get

$$
\begin{aligned}
\phi\left(\nabla_{\xi} \nabla_{\xi} h\right) X= & -\left[\xi(\xi(f))+2 f \xi(f)+\frac{\xi(a+b)}{2}\right] \phi^{2} X \\
& -2 \xi(f) \phi h X-2 f \phi\left(\nabla_{\xi} h\right) X+\left(\nabla_{\xi} h\right) h X+h\left(\nabla_{\xi} h\right) X .
\end{aligned}
$$

Therefore by using (5.3) and (5.4), the formula of Lemma 4.1 becomes

$$
\begin{aligned}
\left(\mathcal{L}_{\xi} R\right)(X, \xi) \xi & =-\frac{\xi(a+b)}{2} \phi^{2} X-\left(\nabla_{\xi} h\right) h X+h\left(\nabla_{\xi} h\right) X \\
& =-\frac{\xi(a+b)}{2} \phi^{2} X+2\left[\xi(f)+f^{2}+\frac{1}{2}(a+b)\right] \phi h X+2 \phi h^{3} X .
\end{aligned}
$$

Combining this with (5.2) yields

$$
0=\left[\xi(f)+f^{2}+\frac{1}{2}(a+b)\right] h X+h^{3} X .
$$


As in the proof of (4.15), the formula (5.5) yields the assertion.

Proof of Theorem 1.3. In view of Proposition 5.1, we know $a+b=-2 \xi(f)-2 f^{2}$. Because $\left(\mathcal{L}_{V} g\right)(Y, Z)=2 \lambda g(Y, Z)-2 g(Q Y, Z)$ for any vector fields $Y, Z$, we compute

$$
\begin{aligned}
& \left(\nabla_{X} \mathcal{L}_{V} g\right)(Y, Z)=-2 g\left(\left(\nabla_{X} Q\right) Y, Z\right) \\
= & -2 g(X(a) Y+X(b) \eta(Y) \xi+b g(A X, Y) \xi+b \eta(Y) A X, Z) .
\end{aligned}
$$

Hence

$$
\begin{aligned}
g\left(\left(\mathcal{L}_{V} \nabla\right)(Y, Z), X\right)= & \frac{1}{2}\left\{\left(\nabla_{Z} \mathcal{L}_{V} g\right)(Y, X)+\left(\nabla_{Y} \mathcal{L}_{V} g\right)(Z, X)-\left(\nabla_{X} \mathcal{L}_{V} g\right)(Y, Z)\right\} \\
= & -\{g(Z(a) Y+Z(b) \eta(Y) \xi+b g(A Z, Y) \xi+b \eta(Y) A Z, X) \\
& +g(Y(a) Z+Y(b) \eta(Z) \xi+b g(A Y, Z) \xi+b \eta(Z) A Y, X) \\
& -g(X(a) Y+X(b) \eta(Y) \xi+b g(A X, Y) \xi+b \eta(Y) A X, Z)\} \\
= & -\{Z(a) g(Y, X)+Z(b) \eta(Y) \eta(X)+Y(a) g(X, Z)+Y(b) \eta(Z) \eta(X) \\
& +2 b \eta(X) g(A Y, Z)-X(a) g(Y, Z)-X(b) \eta(Y) \eta(Z)\} .
\end{aligned}
$$

That means that

$$
\begin{aligned}
\left(\mathcal{L}_{V} \nabla\right)(Y, Z) & =-Z(a) Y-Z(b) \eta(Y) \xi-Y(a) Z-Y(b) \eta(Z) \xi \\
& -2 b g(A Y, Z) \xi+g(Z, Y) \nabla a+\eta(Y) \eta(Z) \nabla b .
\end{aligned}
$$

Taking the covariant differentiation of $\left(\mathcal{L}_{V} \nabla\right)(Y, Z)$ along any vector field $X$, we may obtain

$$
\begin{aligned}
& \left(\nabla_{X} \mathcal{L}_{V} \nabla\right)(Y, Z) \\
& =-g\left(Z, \nabla_{X} \nabla a\right) Y-g\left(Z, \nabla_{X} \nabla b\right) \eta(Y) \xi-Z(b) g(A X, Y) \xi \\
& -Z(b) \eta(Y) A X-g\left(Y, \nabla_{X} \nabla a\right) Z-g\left(Y, \nabla_{X} \nabla b\right) \eta(Z) \xi-Y(b) g(A X, Z) \xi \\
& -Y(b) \eta(Z) A X-2 X(b) g(A Y, Z) \xi-2 b g\left(\left(\nabla_{X} A\right) Y, Z\right) \xi-2 b g(A Y, Z) A X \\
& +g(Z, Y) \nabla_{X} \nabla a+g(A X, Y) \eta(Z) \nabla b+\eta(Y) g(A X, Z) \nabla b+\eta(Y) \eta(Z) \nabla_{X} \nabla b .
\end{aligned}
$$

Thus by virtue of (3.8) we have

$$
\begin{aligned}
& \left(\mathcal{L}_{V} R\right)(X, Y) Z \\
= & \left(\nabla_{X} \mathcal{L}_{V} \nabla\right)(Y, Z)-\left(\nabla_{Y} \mathcal{L}_{V} \nabla\right)(X, Z) \\
= & -g\left(Z, \nabla_{X} \nabla a\right) Y-g\left(Z, \nabla_{X} \nabla b\right) \eta(Y) \xi-Z(b) \eta(Y) A X-Y(b) g(A X, Z) \xi \\
& -Y(b) \eta(Z) A X-2 X(b) g(A Y, Z) \xi-2 b g\left(\left(\nabla_{X} A\right) Y, Z\right) \xi-2 b g(A Y, Z) A X \\
& +g(Z, Y) \nabla_{X} \nabla a+\eta(Y) g(A X, Z) \nabla b+\eta(Y) \eta(Z) \nabla_{X} \nabla b \\
& -\left[-g\left(Z, \nabla_{Y} \nabla a\right) X-g\left(Z, \nabla_{Y} \nabla b\right) \eta(X) \xi-Z(b) \eta(X) A Y-X(b) g(A Y, Z) \xi\right. \\
& -X(b) \eta(Z) A Y-2 Y(b) g(A X, Z) \xi-2 b g\left(\left(\nabla_{Y} A\right) X, Z\right) \xi-2 b g(A X, Z) A Y \\
& \left.+g(Z, X) \nabla_{Y} \nabla a+\eta(X) g(A Y, Z) \nabla b+\eta(X) \eta(Z) \nabla_{Y} \nabla b\right]
\end{aligned}
$$

since $g\left(X, \nabla_{Y} \nabla \zeta\right)=g\left(Y, \nabla_{X} \nabla \zeta\right)$ for any function $\zeta$ and vector fields $X, Y$ followed from Poincare lemma. 
By contracting over $X$ in the previous formula, we have

$$
\begin{aligned}
& \left(\mathcal{L}_{V} \operatorname{Ric}\right)(Y, Z) \\
= & g\left(Z, \nabla_{Y} \nabla a\right)-g\left(Z, \nabla_{\xi} \nabla b\right) \eta(Y)-2 f Z(b) \eta(Y) \\
& -2 f Y(b) \eta(Z)-2 \xi(b) g(A Y, Z)-2 b g\left(\left(\nabla_{\xi} A\right) Y, Z\right)-4 f b g(A Y, Z) \\
& +g(Z, Y) \Delta a+\eta(Y) g(A \nabla b, Z)+\eta(Y) \eta(Z) \Delta b \\
& -\left[-g\left(Z, \nabla_{Y} \nabla b\right)-A Y(b) \eta(Z)+\eta(Z) g\left(\nabla_{Y} \nabla b, \xi\right)\right] .
\end{aligned}
$$

On the other hand, since $Q X=a X+b \eta(X) \xi$ and $r=3 a+b$, the equation (4.6) is expressed as

$$
\begin{aligned}
R(X, Y) Z= & b g(Y, Z) \eta(X) \xi-b g(X, Z) \eta(Y) \xi+b \eta(Y) \eta(Z) X-b \eta(X) \eta(Z) Y \\
& +\frac{a-b}{2}[g(Y, Z) X-g(X, Z) Y] .
\end{aligned}
$$

Thus we get the Lie derivative of $R(X, Y) Z$ along $V$ from (5.8)

$$
\begin{aligned}
& \left(\mathcal{L}_{V} R\right)(X, Y) Z \\
= & V(b) g(Y, Z) \eta(X) \xi+b\left(\mathcal{L}_{V} g\right)(Y, Z) \eta(X) \xi \\
& +b g(Y, Z)\left(\mathcal{L}_{V} \eta\right)(X) \xi+b g(Y, Z) \eta(X) \mathcal{L}_{V} \xi \\
& -\left[V(b) g(X, Z) \eta(Y) \xi+b\left(\mathcal{L}_{V} g\right)(X, Z) \eta(Y) \xi\right. \\
& \left.+b g(X, Z)\left(\mathcal{L}_{V} \eta\right)(Y) \xi+b g(X, Z) \eta(Y) \mathcal{L}_{V} \xi\right] \\
& +V(b) \eta(Y) \eta(Z) X+b\left(\mathcal{L}_{V} \eta\right)(Y) \eta(Z) X+b \eta(Y)\left(\mathcal{L}_{V} \eta\right)(Z) X \\
& -\left[V(b) \eta(X) \eta(Z) Y+b\left(\mathcal{L}_{V} \eta\right)(X) \eta(Z) Y+b \eta(X)\left(\mathcal{L}_{V} \eta\right)(Z) Y\right] \\
& +\frac{V(a-b)}{2}[g(Y, Z) X-g(X, Z) Y]+\frac{a-b}{2}\left[\left(\mathcal{L}_{V} g\right)(Y, Z) X-\left(\mathcal{L}_{V} g\right)(X, Z) Y\right]
\end{aligned}
$$

Contracting over $X$ in (5.9) gives

$$
\begin{aligned}
& \left(\mathcal{L}_{V} \operatorname{Ric}\right)(Y, Z) \\
= & V(b) g(Y, Z)+b\left(\mathcal{L}_{V} g\right)(Y, Z)+b g(Y, Z)\left(\mathcal{L}_{V} \eta\right)(\xi) \\
& +b g(Y, Z) g\left(\mathcal{L}_{V} \xi, \xi\right)-\left[V(b) \eta(Z) \eta(Y)+b\left(\mathcal{L}_{V} g\right)(\xi, Z) \eta(Y)\right. \\
& \left.+b \eta(Z)\left(\mathcal{L}_{V} \eta\right)(Y)+b \eta(Y) g\left(\mathcal{L}_{V} \xi, Z\right)\right] \\
& +3 V(b) \eta(Y) \eta(Z)+3 b\left(\mathcal{L}_{V} \eta\right)(Y) \eta(Z)+3 b \eta(Y)\left(\mathcal{L}_{V} \eta\right)(Z) \\
& -\left[V(b) \eta(Y) \eta(Z)+b\left(\mathcal{L}_{V} \eta\right)(Y) \eta(Z)+b \eta(Y)\left(\mathcal{L}_{V} \eta\right)(Z)\right] \\
& +\frac{V(a-b)}{2}[2 g(Y, Z)]+\frac{a-b}{2}\left[2\left(\mathcal{L}_{V} g\right)(Y, Z)\right] \\
= & b(Y, Z) g\left(\mathcal{L}_{V} \xi, \xi\right)-\left[b\left(\mathcal{L}_{V} g\right)(\xi, Z) \eta(Y)+b \eta(Y) g\left(\mathcal{L}_{V} \xi, Z\right)\right] \\
& +V(b) \eta(Y) \eta(Z)+b\left(\mathcal{L}_{V} \eta\right)(Y) \eta(Z)+2 b \eta(Y)\left(\mathcal{L}_{V} \eta\right)(Z) \\
& +V(a) g(Y, Z)+a\left(\mathcal{L}_{V} g\right)(Y, Z) \\
= & b g(Y, Z) g\left(\mathcal{L}_{V} \xi, \xi\right)-\left[2 b(\lambda-a-b) \eta(Z) \eta(Y)+b \eta(Y) g\left(\mathcal{L}_{V} \xi, Z\right)\right] \\
& +V(b) \eta(Y) \eta(Z)+b\left[g(A Y, V)+\eta\left(\nabla_{Y} V\right)\right] \eta(Z) \\
& +2 b \eta(Y)\left[g(A Z, V)+\eta\left(\nabla_{Z} V\right)\right]+V(a) g(Y, Z) \\
& +a(2 \lambda g(Y, Z)-2 a g(Y, Z)-2 b \eta(Y) \eta(Z))
\end{aligned}
$$


Finally, by comparing (5.10) with (5.7) we get

$$
\begin{aligned}
& g\left(Z, \nabla_{Y} \nabla a\right)-g\left(Z, \nabla_{\xi} \nabla b\right) \eta(Y)-2 f Z(b) \eta(Y) \\
& -2 f Y(b) \eta(Z)-2 \xi(b) g(A Y, Z)-2 b g\left(\left(\nabla_{\xi} A\right) Y, Z\right)-4 f b g(A Y, Z) \\
& +g(Z, Y) \Delta a+\eta(Y) g(A \nabla b, Z)+\eta(Y) \eta(Z) \Delta b \\
& -\left[-g\left(Z, \nabla_{Y} \nabla b\right)-A Y(b) \eta(Z)+\eta(Z) g\left(\nabla_{Y} \nabla b, \xi\right)\right] \\
= & b g(Y, Z) g\left(\mathcal{L}_{V} \xi, \xi\right)-\left[2 b(\lambda-a-b) \eta(Z) \eta(Y)+b \eta(Y) g\left(\mathcal{L}_{V} \xi, Z\right)\right] \\
& +V(b) \eta(Y) \eta(Z)+b\left[g(A Y, V)+\eta\left(\nabla_{Y} V\right)\right] \eta(Z) \\
& +2 b \eta(Y)\left[g(A Z, V)+\eta\left(\nabla_{Z} V\right)\right]+V(a) g(Y, Z) \\
& +a(2 \lambda g(Y, Z)-2 a g(Y, Z)-2 b \eta(Y) \eta(Z)) .
\end{aligned}
$$

Replacing $Y$ and $Z$ by $\phi Y$ and $\phi Z$ respectively leads to

$$
\begin{aligned}
& g\left(\phi Z, \nabla_{\phi Y} \nabla a\right)-2 \xi(b) g(A \phi Y, \phi Z)-2 b g\left(\left(\nabla_{\xi} A\right) \phi Y, \phi Z\right) \\
& -4 f b g(A \phi Y, \phi Z)+g(\phi Z, \phi Y) \Delta a-\left[-g\left(\phi Z, \nabla_{\phi Y} \nabla b\right)\right] \\
= & b g(\phi Y, \phi Z) g\left(\mathcal{L}_{V} \xi, \xi\right)+V(a) g(\phi Y, \phi Z)+2 a(\lambda-a) g(\phi Y, \phi Z) .
\end{aligned}
$$

Because $\sum_{i=1}^{3}\left(\mathcal{L}_{V}\left(e_{i}, e_{i}\right)\right)=0$ (see [7, Eq.(9)]), by (5.6) we obtain the gradient field

$$
\nabla(a+b)=2[\xi(b)+2 f b] \xi .
$$

Therefore the formula (5.11) can be simplified as

$$
\begin{aligned}
& -2 b g\left(\left(\nabla_{\xi} A\right) \phi Y, \phi Z\right)+g(\phi Z, \phi Y) \Delta a \\
& =b g(\phi Y, \phi Z) g\left(\mathcal{L}_{V} \xi, \xi\right)+V(a) g(\phi Y, \phi Z)+2 a(\lambda-a) g(\phi Y, \phi Z) .
\end{aligned}
$$

Moreover, using (2.5) and (5.3) we compute

$$
\begin{aligned}
\left(\nabla_{\xi} A\right) \phi Y & =-\xi(f) \phi^{3} Y+\phi^{2}\left(\nabla_{\xi} h\right) Y \\
& =\xi(f) \phi Y+\left(\frac{a+b}{2}+\xi(f)+f^{2}\right) \phi Y+2 f h Y+\phi h^{2} Y \\
& =\left(\frac{a+b}{2}+2 \xi(f)+f^{2}\right) \phi Y+2 f h Y+\phi h^{2} Y .
\end{aligned}
$$

Substituting this into (5.13) yields

$$
\begin{aligned}
- & 2 b g\left(2 f h Y+\phi h^{2} Y, \phi Z\right) \\
= & \left\{b g\left(\mathcal{L}_{V} \xi, \xi\right)+2 b\left[\frac{a+b}{2}+2 \xi(f)+f^{2}\right]\right. \\
& -\Delta a+V(a)+2 a(\lambda-a)\} g(\phi Y, \phi Z) .
\end{aligned}
$$

Replacing $Z$ by $\phi Y$ in (5.14) gives

$$
0=b g\left(2 f h Y+\phi h^{2} Y, \phi^{2} Y\right)=-b g\left(2 f h Y+\phi h^{2} Y, Y\right) .
$$

Next we divide into two cases.

Case I: $h=0$. Then the Eq.(2.8) and Proposition 5.1 imply $\xi(f)=0$, i.e., $f$ is constant as $\nabla f=\xi(f) \xi$ followed from $d f \wedge \eta=0$, so $M$ is an $\alpha$-cosymplectic manifold.

Case II: $h \neq 0$. Suppose that $Y=e$ is an unit eigenvector corresponding to the nonzero eigenvalue $\lambda^{\prime}$ of $h$, then we may obtain from (5.15) that $f b=0$. If the function $b$ is not zero, there is an open neighborhood $\mathcal{U}$ such that $\left.b\right|_{\mathcal{U}} \neq 0$, so $\left.f\right|_{\mathcal{U}}=0$. By Proposition 5 .1 it implies $\left.(a+b)\right|_{\mathcal{U}}=0$. Notice that trace $\left(h^{2}\right)=-(a+b)-2\left(\xi f+f^{2}\right)$, so we have trace $\left.\left(h^{2}\right)\right|_{\mathcal{U}}=0$, 
i.e., $\left.h\right|_{\mathcal{U}}=0$. It comes to a contradiction, so $b=0$ and $a$ is constant by (5.12). Hence by (1.3) we obtain Ric $=a g$, where $a=-2 \xi(f)-2 f^{2}$. Moreover, we know $\lambda=a$ or $a=0$ by

(5.13). Finally we show $a \neq 0$. In fact, if $a=0$ then $\xi(f)=-f^{2}$. Substituting this into (2.8) we find trace $\left(h^{2}\right)=-2\left(\xi(f)+f^{2}\right)=0$, which is impossible because $h \neq 0$.

Thus we obtain from (5.8)

$$
R(X, Y) Z=\frac{\lambda}{2}(g(Y, Z) X-g(X, Z) Y) .
$$

Summing up the above two cases we finish the proof Theorem 1.3 .

\section{Acknowledgement}

The author would like to thank the referee for the comments and valuable suggestions.

\section{REFERENCES}

[1] N. Aktan, M. Yildirim, C. Murathan, Almost f-cosymplectic manifolds, Mediterr. J. Math. 11(2014), 775-787.

[2] J. T. Cho, Ricci solitons in almost contact geometry. Proceedings of the 17th International Workshop on Differential Geometry [Vol. 17], 8595, Natl. Inst. Math. Sci. (NIMS ), Taejon, 2013.

[3] J. T. Cho, Almost contact 3-manifolds and Ricci solitons, Int. J. Geom. Methods Mod. Phys. 10(2012), 515-532.

[4] H. Endo, Non-existence of almost cosymplectic manifolds satisfying a certain condition, Tensor(N.S). 63 (2002), 272-284.

[5] M. Fernández, E. García-Río, A Remark on compact Ricci solitons, Math. Ann. 340(2008), 893-896

[6] D. Friedan, Nonlinear models in $2+\epsilon$ dimensions, Ann Phys. 163(1985), 318-419.

[7] A. Ghosh, Kenmotsu 3-metric as a Ricci soliton, Chaos, Solitons Fractals, 44(2011), 647-650.

[8] A. Ghosh, R. Sharma, Sasakian metric as a Ricci soliton and related results, J. Geom. Phys. 75(2013), $1-6$.

[9] A. Ghosh, R. Sharma, K-contact metrics as Ricci solitons, Beitr Algebra Geom. 53(2012), 25-30.

[10] S. I. Goldberg, K. Yano, Integrability of almost cosymplectic structure, Pacific. J. Math. 31(1969), 373-382.

[11] T. W. Kim, H. K. Pak, Canonical foliations of certain classes of almost contact metric structres, Acta Math. Sinica Eng. Ser. Aug. 21(2005), 841-846.

[12] B. Cappelletti-Montano, A. De Nicola, I. Yudin, A survey on cosymplectic geometry, Rev. Math. Phys. 25(2013), 2068-2078.

[13] O. Munteanu, N. Sesum, On Gradient Ricci Solitons, J. Geom. Anal. 23(2013), 539-561.

[14] H. Öztürk, N. Aktan, C. Murathan, Almost $\alpha$-cosymplectic $(\kappa, \mu, \nu)$-spaces, arXiv:1007.0527v1.

[15] P. Petersen, W. Wylie, Rigidity of gradient Ricci solitons, Pacific. J. of Math. 241(2009), 329-345.

[16] R. Sharma, Certain results on K-contact and $(\kappa, \mu)$-contact manifolds, J. Geom. 89(2008), 138-147.

[17] Y. Wang, A generalization of the Goldberg conjecture for coKähler manifolds, Mediterr. J. Math. 13(2016), 2679-2690.

[18] Y. Wang, X. Liu, Ricci solitons on three dimensional $\eta$-Einstein almost Kenmotsu manifolds, Taiwanese. J. Math. 19(2015), 91-100.

[19] Y. Wang, U. C. De, X. Liu, Gradient Ricci solitons on almost Kenmotsu manifolds, Publication. de l'Institut Math. 98(2015), 277-235.

[20] K. Yano, Integral Formula in Riemannian Geometry. Macrcel Dekker, New York, (1970).

College of Science, China University of Petroleum-Beijing, Beijing, 102249, China

E-mail address: xmchen@cup.edu.cn 